Muñoz-Cantero, J., Rebollo, N. \& Espiñeira, E.M. (2014). Percepción de competencias en el EEES: análisis en el Grado de Educación Primaria. Revista Electrónica Interuniversitaria de Formación del Profesorado, 17 (3), 123-139.

DOI: http://dx.doi.org/10.6018/reifop.17.3.204091

\title{
Percepción de competencias en el EEES: análisis en el Grado de Educación Primaria
}

\author{
Jesús Miguel Muñoz Cantero, Nuria Rebollo Quintela, Eva María Espiñeira Bellón \\ Universidad de A Coruña
}

\section{Resumen}

El desarrollo de los títulos de acuerdo al Espacio Europeo de Educación Superior (EEES) exige el diseño de sus títulos con el fin de que el alumnado adquiera una serie de competencias específicas y generales que, en algunos casos, como en el grado en Educación Primaria están definidas de acuerdo a la legislación pertinente. Corresponde a las universidades lograr que este alumnado las adquiera. Finalizada la implantación de éstos títulos y una vez que ha salido la primera promoción de acuerdo al EEES corresponde hacer un análisis y reflexionar sobre cómo éstas se están adquiriendo. En este artículo se analiza la percepción de competencias por parte 55 estudiantes de último curso de Grado en Educación Primaria en base a tres dimensiones: desarrollo, dominio y relevancia otorgados. Los resultados indican un alto grado de satisfacción, siendo más elevados los referidos al grado de relevancia otorgado a cada una de las competencias. Les siguen las valoraciones referidas al grado de dominio y finalmente al desarrollo. Específicamente se evidenció que aquellos ítems relativos a competencias en contextos multilingües o capacidad de expresión oral y escrita en varias lenguas (al menos en una lengua extranjera) obtienen resultados inferiores.

\section{Palabras clave}

Competencias; Espacio Europeo de Educación Superior; Educación Primaria; Metodologías docentes.

\section{Perception of competencies in the EHEA: analysis in the Primary Education Degree}

Contacto

Jesús Miguel Muñoz Cantero, jesus.miguel.munoz@udc.es, Universidad de A Coruña, Facultad de Ciencias de la Educación, Campus de Elviña, s/n, CP 15071 


\begin{abstract}
The development of titles according to the European Higher Education Area (EHEA) requires the design of their titles so the students acquire a series of specific and generals competencies are defined according to the relevant legislation as in the Primary Education degree. It corresponds to the universities achieve this students to acquire them. After the implementation of these titles and once it has left the first class according to the EHEA corresponds to do an analysis and reflect on how they are acquiring. This article discusses the perception of competence by part 55 students of final year degree in Primary Education based on three dimensions: development, domain and importance granted. The results indicate a high degree of satisfaction, being higher than those related to the degree of importance given to each of the competencies. Follow les estimations related to the degree of domain and finally to the development. Specifically showed lower results to be obtained by those items relating to competences in multilingual contexts or capacity of speaking and writing in several languages (at least in a foreign language).
\end{abstract}

\title{
Key words
}

Competency; European Higher Education; Education; Teaching methodologies.

\section{Introducción}

La Ley orgánica 2/2006, de 3 de mayo, de educación, establece en su artículo 93, que la profesión de Maestro en Educación Primaria se conformará como profesión regulada, cuyo ejercicio requiere estar en posesión del título oficial de Grado obtenido de acuerdo a lo previsto en el RD 1393/2007, de 29 de octubre, por el que se establece la ordenación de las enseñanzas universitarias oficiales.

La propuesta efectuada por la Agencia Nacional de Evaluación de la Calidad y Acreditación (ANECA, 2005, p. 219) para el diseño de los estudios de Grado en Educación Primaria presentaba las siguientes características:

Se propone un Grado de Maestro de Educación Primaria (240 créditos ECTS) que debe conferir a los titulados, competencias docentes generales para ayudar al desarrollo, tutelar el aprendizaje y promover la consecución de los objetivos que establece el Sistema Educativo para la Educación Primaria. Ha de ser capaz de ser responsable docente de todas las materias comunes que actualmente son competencia de los tutores (Matemáticas, Lengua, Ciencias-Geografía e Historia [o Conocimiento del Medio] y Ed. Artística [plástica]). Además habrá de disponer de competencias docentes específicas en uno de estos ámbitos (...): Ed. Física, Ed. Musical, Lengua Extranjera o Atención a Necesidades Educativas Especiales.

Tomando esta propuesta, la mayoría de las universidades españolas han optado por diversos itinerarios formativos para el Grado en Educación Primaria, recogiendo las especialidades de los antiguos estudios de Maestro/a. Esta opción, ha condicionado también el desarrollo de las competencias a adquirir por los/las titulados/as, de acuerdo a las señaladas en la Orden $\mathrm{ECI} / 3857 / 2007$, de 27 de diciembre, por la que se establecen los requisitos para la verificación de los títulos universitarios oficiales que habiliten para el ejercicio de la profesión de Maestro en Educación Primaria.

Atendiendo al anterior contexto legislativo, en el presente artículo se desarrolla el diseño de los estudios de Grado en Educación Primaria en la Facultad de Ciencias de la Educación de la Universidad de A Coruña. A continuación, se profundiza en las competencias propuestas en este Grado. 
En la parte metodológica del presente estudio, se realiza un análisis comparativo a través de la percepción del alumnado atendiendo al grado de desarrollo, dominio y relevancia otorgada a cada una de las competencias del Grado. Este análisis se justifica teniendo en cuenta que una de las metas que deben plantearse las universidades es la de valorar el nivel de competencias alcanzado por el alumnado de acuerdo con los programas de formación recibidos.

\section{Los estudios de Grado en Educación Primaria en la Universidad de A Coruña}

\section{Objetivos y planificación de las enseñanzas}

El 20 de junio de 2008 se aprueba la propuesta de título de Grado en Educación Primaria por la Universidad de A Coruña.

En dicha propuesta, atendiendo a lo señalado en el apartado anterior, se establece un título de Grado de 240 créditos ECTS, con tres itinerarios formativos o menciones (Educación Especial, Educación Física y Especialización Curricular). Las dos primeras menciones se eligen teniendo en cuenta que con el presente título se extinguen las titulaciones de Maestro en Educación Física y Maestro en Audición y Lenguaje. Con la tercera mención se pretende ofrecer la posibilidad de profundizar en algunos aspectos de la formación didáctica-disciplinar del Grado.

A continuación, en dicha memoria se hace referencia a los objetivos generales del título y competencias.

En un primer momento, teniendo en cuenta el apartado 3 (Objetivos) de la ya mencionada Orden $\mathrm{ECl} / 3857 / 2007$, se recogen las 12 competencias que el alumnado de dicho Grado debe adquirir, competencias no analizadas en el presente trabajo debido a que se han entendido en la memoria de verificación como objetivos.

En segundo lugar, se establece una doble clasificación de competencias: específicas y transversales/genéricas, aunque es de destacar que, dentro de estas últimas, se contemplan también las competencias nucleares, definidas concretamente en la Universidad de $\mathrm{A}$ Coruña, como veremos en el siguiente apartado. No se redactan dichas competencias, teniendo en cuenta que son las que se establecen en el instrumento de recogida de información (ver anexo).

\section{Estudio de las competencias del Grado}

El término de competencia ha tenido "variadas interpretaciones y aplicaciones prácticas" (Pavié, 2011, p. 69) tanto en el ámbito educativo como en el empresarial. No obstante, Bautista-Cerro (2006) establece que mientras en el ámbito educativo se refieren a conocimientos, capacidades, actitudes y conceptos afines, en el ámbito empresarial, a aptitudes, capacidades, productividad, etc.

Desde la perspectiva educativa de este trabajo, es necesario señalar que existen, diferentes concepciones en torno al término de competencia (Cano, 2008; Carreras y Perrenoud, 2008; Riesco, 2008; Sobrado, 2010) y a sus posibles tipologías o clasificaciones (Alex, 1991; Bunk, 1994; Echeverría, 2005; Le Boterf, 1993).

En cuanto al término, a pesar de no contar con un acuerdo unánime, la mayoría de las definiciones se centran en "el nivel de capacitación, conocimientos, saberes, actitudes, capacidades y habilidades que tienen las personas y que dominan o deben dominar" (Muñoz Cantero, Rebollo Quintela y Espiñeira Bellón, 2013, pp. 228-229). 
En cuanto a las tipologías, la mayoría de los autores y autoras se han decantado por una doble clasificación de competencias: genéricas o transversales y específicas (Albert Gómez, 2006; Bedolla y García, 2009; Borrero y Contreras, 2009; Corominas et al., 2006; García Fraile, 2008; Proyecto Tunning, 2003). En la Universidad de A Coruña, el título de Grado en Educación Primaria, también se ha acogido a dicha clasificación.

En este sentido, haciendo referencia al ámbito universitario, las competencias genéricas de este título se entienden como aquellas competencias comunes y exigidas en todas las materias de la titulación y se complementan con las denominadas competencias nucleares (definidas en la Universidad de A Coruña), competencias comunes a la mayoría de las titulaciones.

Las competencias específicas son entendidas como aquellas propias de un ámbito o titulación, orientadas a la consecución de un perfil específico del graduado o graduada, o las que se pretenden fomentar y desarrollar en cada asignatura.

Es preciso destacar que la ejecución efectiva del Espacio Europeo de Educación Superior debe proporcionar una formación universitaria en la que se integren competencias básicas, generales y específicas, atendiendo a lo indicado por el Ministerio de Educación, Cultura y Deporte (MECD, 2003). De hecho, otros autores, como Echeverría (2008) hacen referencia a las competencias básicas, competencias que no se recogen en la memoria de verificación inicial de este título y que por tanto, no han sido analizadas.

\section{Relación entre metodologías docentes y competencias}

El cambio que se ha producido en el diseño de los procesos formativos basados en competencias, ha ocasionado un cambio en el modelo de enseñanza-aprendizaje y en el proceso de evaluación de dicho modelo. Ello ha afectado a las diferentes metodologías que han de poner en marcha alumnado y profesorado (Belinchón Romo, de Diego Álvarez y Velasco González, 2011).

Con respecto al profesorado, ha tenido que modificar las metodologías docentes y aplicar nuevas metodologías evaluativas para la adquisición de dichas competencias, entendiendo que es necesario reforzar la modificación del papel del alumnado que ya no debe exclusivamente centrarse en la adquisición de conocimientos sino como impulsor de su aprendizaje a lo largo de toda la vida (Delgado, Borge, García, Oliver y Salomón, 2005; Montero, 2010). El alumnado, por su parte, ha tenido que modificar las técnicas de aprendizaje para la transmisión del conocimiento.

Las metodologías didácticas, como señala Fernández March (s.f.), constituyen uno de los componentes de los proyectos formativos y debido a que han ido avanzando con el paso del tiempo, existen numerosos listados que las catalogan. En este sentido, en el plan formativo del Grado en Educación Primaria se recogen cuarenta metodologías docentes que cada profesor/a valorará para su elección como actividad docente, metodología a seguir o criterio de evaluación. Dependiendo del tipo de metodología elegido, la enseñanza se desarrollará a través de distintos grados de participación del profesorado y del alumnado.

Las competencias genéricas de este título, van en la línea de aprender a aprender, resolver problemas, trabajar de forma colaborativa,... que supuestamente se deben desarrollar mediante la aplicación de estas metodologías docentes. 


\section{Metodología}

Para llevar a cabo esta investigación se han planteado los siguientes objetivos:

- Verificar qué competencias son las mejor y peor valoradas por el alumnado, de las definidas en el Plan de Estudios de la titulación del Grado en Educación Primaria.

- Evidenciar si existen diferencias estadísticamente significativas entre las dimensiones analizadas: desarrollo, dominio y relevancia.

- Proponer acciones orientadas a la mejora.

\section{Participantes}

En el estudio ha participado alumnado de último curso del Grado en Educación Primaria de la Facultad de Ciencias de la Educación de A Coruña. Se ha realizado un muestreo no probabilístico intencional, dado que se ha seleccionado específicamente un título concreto y al alumnado que cumpliera la característica de haber realizado el $2^{\circ}$ período de Practicum.

La muestra de estudiantes del Grado en Educación Primaria se compone de 55 sujetos ( $\mathrm{N}=$ 91) donde el $94.55 \%$ son mujeres y el $5.45 \%$ (figura 1 ). Nos encontramos con una muestra sesgada feminizada, dado que el perfil de ingreso en las titulaciones de educación es mayoritariamente femenino.

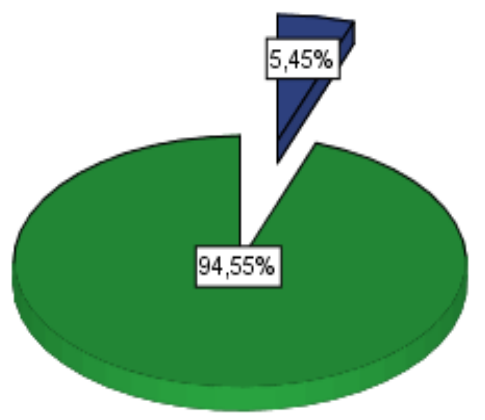

Masculino

Femenino

Figura 1. Distribución de la variable sexo en el Grado de Educación Primaria

En el Grado en Educación Primaria, la distribución muestral de la variable edad oscila entre 21 y 40 años, siendo la moda de edad los 21 años (38.9\%).

\section{Instrumento}

En el curso 2012-2013 se elaboró un cuestionario que recogía las competencias definidas en el plan de estudios de la titulación del Grado de Educación Primaria de la Facultad de Ciencias de la Educación de la Universidad de A Coruña. Consideramos que la presentación de los ítems puede ser extensa, ya que se ha conservado la redacción de las competencias originales para evitar introducir algún sesgo debido a la manipulación en la expresión de las mismas.

Este instrumento está estructurado en cuatro partes:

- Datos personales (curso, edad, sexo)

- Competencias nucleares (8 ítems)

- Competencias específicas (66 ítems)

- Competencias transversales (20 ítems) 
El instrumento elaborado nos permite extraer información sobre la percepción que el alumnado tiene de las competencias, de acuerdo a las siguientes dimensiones:

- Grado de desarrollo: pretendemos analizar si se ha incidido en ellas a lo largo de la titulación

- Grado de dominio: percepción del alumnado sobre el grado de adquisición que posee de las competencias definidas en el título

- Grado de relevancia: importancia que les concede para su futuro profesional

La valoración que el alumnado otorga, a las competencias, se ha realizado en base a una escala Likert de 5 opciones de respuestas, siendo 1 nada y 5 mucho.

\section{Procedimiento}

Para dar respuesta a los objetivos planteados en la investigación se ha utilizado la estadística descriptiva e inferencial, cuyo tratamiento se ha efectuado a través del paquete estadístico SPSS 20. En primer lugar se calcula la consistencia interna del instrumento diseñado mediante el índice de fiabilidad a de Cronbach, posteriormente, se realiza un análisis de las medidas de tendencia central y de dispersión (media y desviación típica). Finalmente, se procede a un análisis comparativo con la prueba Friedman para K muestras relacionadas a fin de establecer si existen diferencias estadísticamente significativas entre las 3 dimensiones analizadas.

\section{Resultados}

- Análisis para de Grado de Educación Primaria

\section{- Fiabilidad del instrumento}

Para determinar la consistencia interna del instrumento diseñado, se examinó la fiabilidad del cuestionario compuesto por 94 ítems, para cada una de sus dimensiones: desarrollo, dominio y relevancia. Los resultados muestran una alta fiabilidad (Maroco y GarcíaMarques, 2006; McMillan y Schumacher, 2005) ya que el a de Cronbach es superior a .9 en todos los casos, como muestra la tabla 1.

Tabla 1. Fiabilidad del instrumento en la dimensión desarrollo, dominio y relevancia

\begin{tabular}{cccc}
\hline $\mathrm{N}^{\circ}$ de elementos & $\begin{array}{c}\text { Alfa de Cronbach } \\
\text { Dimensión } \\
\text { desarrollo }\end{array}$ & $\begin{array}{c}\text { Alfa de Cronbach } \\
\text { Dimensión } \\
\text { dominio }\end{array}$ & $\begin{array}{c}\text { Alfa de Cronbach } \\
\text { Dimensión } \\
\text { relevancia }\end{array}$ \\
\hline 94 & .980 & .979 & .976 \\
\hline
\end{tabular}

\section{- Análisis descriptivo de las competencias nucleares}

Los datos obtenidos muestran que, de las 8 competencias nucleares definidas en el título de Grado en Educación Primaria, son consideradas de gran relevancia y evidencian dominarlas, ya que los valores de la media son superiores a 3. Sin embargo, el alumnado percibe que las competencias "Dominar la expresión y la comprensión de forma oral y escrita de un idioma extranjero" ( $\overline{\mathrm{x}}=2.35)$ y "Entender la importancia de la cultura emprendedora y conocer los medios al alcance de las personas emprendedoras" ( $\bar{x}=2.15)$ han sido vagamente tratadas en su plan de estudios. 


\section{- Análisis descriptivo de las competencias específicas}

El alumnado percibe haber desarrollado la casi totalidad de las 66 competencias específicas (medias superiores a 2.5), a excepción de algunas relativas a habilidades comunicativas y relacionales "Mostrar habilidades sociales para entender a las familias y hacerse entender por ellas" ( $\overline{\mathrm{x}}=1.98)$ "Conocer las dificultades para el aprendizaje de las lenguas oficiales de estudiantes de otras lenguas" $(\overline{\mathrm{x}}=1.91)$ "Afrontar situaciones de aprendizaje de lenguas en contextos multilingües" ( $\overline{\mathrm{x}}=1.80)$. También puntúan bajo las relacionadas con los sistemas de gestión de la calidad de un centro educativo "Participar en la definición del proyecto educativo y en la actividad general del centro atendiendo a criterios de gestión de calidad" $(\bar{x}=2.29)$, "Participar en las propuestas de mejora en los distintos ámbitos de actuación que se puedan establecer en un centro" $(\overline{\mathrm{x}}=2.24)$ "Conocer y aplicar metodologías y técnicas básicas de investigación educativa y ser capaz de diseñar proyectos de innovación identificando indicadores de evaluación" ( $\overline{\mathrm{x}}=2.29)$.

De igual modo, consideran tener un alto dominio de las competencias definidas en el plan de estudios de Educación Primaria, a excepción de en aquellas que han desarrollado en menor medida.

La importancia concedida a todas las competencias es elevada, con medias superiores a 4, menos en los ítems referidos a los mecanismos de gestión de calidad.

\section{- Análisis descriptivo de las competencias transversales}

Los datos muestran que el alumnado percibe haber desarrollado en gran medida las competencias transversales, a excepción, nuevamente de las relacionadas con habilidades comunicativas "Lectura e interpretación de imágenes" $(\bar{x}=2.25)$ y "Capacidad de expresión oral y escrita en varias lenguas (al menos en una lengua extranjera)" ( $\bar{x}=2.35)$. Los resultados son coincidentes con el estudio de Pazo Haro y Tejada Mora (2012), en el que el alumnado de Educación Física evalúa las competencias definidas en su plan de estudios.

En cuanto a la dimensión dominio, destacan las elevadas puntuaciones otorgadas a los ítems en competencias colaborativas "Trabajar de forma colaborativa" ( $\bar{x}=4.29)$ y "Capacidad para trabajar en equipo de forma cooperativa, para organizar y planificar el trabajo, tomando decisiones y resolviendo problemas, tanto de forma conjunta como individual" ( $\overline{\mathrm{x}}=4.10)$. Martín y Puig (2007) destacan las ventajas de dominar competencias del trabajo colaborativo ya que mediante este se comparten responsabilidades, aumenta la reflexión y el análisis colectivo, se fomenta las intervenciones más globales,...

\section{- Análisis de varianza por rangos de Friedman}

Para evidenciar si las diferencias entre las puntuaciones otorgadas por el estudiante en la dimensión relevancia respecto al dominio y desarrollo son sólo diferencias de medias y no estadísticamente significativas, se ha procedido a realizar un análisis de Friedman.

Los resultados obtenidos mediante esta prueba no paramétrica para $\mathrm{k}$ muestras relacionadas, permiten corroborar que existen diferencias estadísticamente significativas entre las dimensiones desarrollo, dominio y relevancia, con un nivel de significación de $p<$ .05 .

\section{Discusión y conclusiones}

La implantación del Espacio Europeo de Educación Superior ha supuesto la transformación de los estudios de grado en cuanto a su formulación (Pazo Haro y Tejada Mora, 2012), pero 
esta ha de venir ligada al impulso de nuevas metodologías docentes que favorezca el desarrollo y adquisición de las competencias definidas en los títulos.

El mercado laboral global al que el alumnado accede tras su formación, demanda profesionales capaces en determinadas competencias generales: comunicativas, colaborativas, de autorregulación,... Una de las competencias más demandadas y ante la que los graduados españoles consideran poseer un dominio inferior respecto al resto de los graduados europeos es la relacionada con el conocimiento de idiomas extranjeros (Mora, 2003). Aspecto que se pudo evidenciar en nuestra investigación, siendo los ítems "Afrontar situaciones de aprendizaje de lenguas en contextos multilingües" y "Capacidad de expresión oral y escrita en varias lenguas (al menos en una lengua extranjera)" los que obtenían medias inferiores. Resulta interesante este aspecto pues siendo una titulación en la que cada vez más se le exige para su inserción laboral el dominio de lenguas extranjeras, el dominio de éstas no es acorde a lo demandado y aunque los centros o escuelas de idiomas que las universidades ponen en marcha como actividades complementarias que el estudiante puede realizar, el hecho es que son pocos los estudiantes que realizan cursos de idiomas. Podría considerarse la necesidad de incorporar más materias en los títulos de grado que se impartiesen en toras lenguas.

Así mismo, "aprender por competencias debería fomentar el aprendizaje colaborativo para que los estudiantes aprendan a trabajar en equipo" (González Geraldo, del Rincón Igea, Bonilla Sánchez y Sáez López, 2012, p. 192) a través de metodologías docentes como: juego de rol, estudio de casos, debate, resolución de problemas,... competencias señaladas por el alumnado con un alto grado de dominio y relevancia.

Dada la problemática de inserción laboral existente en nuestro país se hace necesario profundizar y desarrollar competencias orientadas a fomentar la cultura emprendedora, no en vano otra de las competencias peor valoradas hace referencia a "Entender la importancia de la cultura emprendedora y conocer los medios al alcance de las personas emprendedoras" sino en

Consideramos necesario realizar un análisis de adquisición de competencias comparando las menciones de especialización curricular establecidas para el título de Grado en Educación Primaria y ampliar el análisis a otras universidades a fin de diagnosticar si se dan las mismas problemáticas diagnosticadas en nuestro centro.

\section{Referencias}

Agencia Nacional de Evaluación de la Calidad y Acreditación (ANECA) (2005). Libro Blanco. Título de Grado en Pedagogía y Educación Social. Madrid: Autor. Recuperado de http://www.aneca.es/var/media/150392/libroblanco_pedagogia1_0305.pdf

Albert Gómez, M.J. (2006). Competencias y sociedad del conocimiento. En M.A. Murga y M.P. Quicios (Coords.), La reforma de la universidad: cambios exigidos por la nueva Europa (pp. 115-122). Madrid: Dykinson.

Alex, L. (1991). Descripción y registro de cualificaciones. El concepto de cualificación. Formación profesional, 2, 23-27.

Bautista-Cerro, M.J. (2006). Educación y competencias. En M.A. Murga y M.P. Quicios (Coords.), La reforma de la universidad: cambios exigidos por la nueva Europa (pp. 123-134). Madrid: Dykinson. 
Bedolla, C. y García, E. (Agosto, 2009). Las competencias genéricas en la Educación Superior y el mercado de trabajo. Ponencia presentada al XI Encuentro Universitario de Actualización Docente, Morelia, Michigan.

Belinchón Romo, M.R., de Diego Álvarez, D. y Velasco González, M. (2011). Nuevas metodologías docentes aplicadas en el aula. El aprendizaje cooperativo y el aprendizaje colaborativo. El trabajo en grupo. Recuperado de http://dugidoc.udg.edu/bitstream/handle/10256/3610/129.pdf?sequence $=1$

Borrero, C. y Contreras, L.C. (2009).Evaluación de competencias en entornos virtuales. En J. I. Aguaded y M.C. Fonseca (Ed.), Huellas de innovación docente en las aulas universitarias (pp.77-86). La Coruña: Netbiblo.

Bunk, G.P. (1994). La trasmisión de las competencias en la formación y perfeccionamiento de profesionales de la RFA. Revista Europea de Formación Profesional, 1, 8-14.

Cano, M.E. (2008). La evaluación por competencias en la educación superior. Profesorado, Revista de currículum y formación del profesorado, 12(3), 1-16. Recuperado de http://www.ugr.es/local/recfpro/rev123COL1.pdf

Carreras, J. y Perrenoud, P. (2008). El debate sobre las competencias en la enseñanza universitaria. Barcelona: ICE y Ediciones Octaedro.

Corominas, E., Tesouro, M., Capell, D., Teixidó, J., Pèlach. J. \& Cortada, R. (2006). Percepciones del profesorado ante la incorporación de las competencias genéricas en la formación universitaria. Revista de Educación, 341, 301-336.

Delgado, A.M., Borge, R., García, J., Oliver, R. y Salomón, L. (2005). Competencias y diseño de la evaluación continua y final en el Espacio Europeo de Educación Superior. Madrid: Ministerio de Educación y Ciencia.

Echeverría, B. (2005). Competencia de acción de los profesionales de la orientación. Madrid: ESIC.

Echeverría, B. (Coord) (2008). Orientación profesional. Barcelona: UOC.

Fernández March, A. (s.f.). Nuevas metodologías docentes. Universidad Politécnica de Valencia. Recuperado

de: http://www.google.es/url?sa=t\&rct=j\&q=\&esrc=s\&source=web\&cd=1\&ved=oCCUQFj AA\&url=http $\% 3$ A\%2F\%2Fwww.usal.es\%2F ofeees\%2FNUEVAS_METODOLOGIAS\%2Fnu evas metodologias docentes.doc\&ei=tayU_DoA5O00QW8g4GQDA\&usg=AFQjCNGqC4HKyZIITgtVCAgGHqR800EMnw\&b $\mathrm{vm}=\mathrm{bv} .70138588$,bs.1,d.ZGU

García Fraile, J.A. (2008). Las competencias en la Educación Superior: calidad y pensamiento complejo. En J.A. García Fraile y S. Tobón (Coords.), Gestión del curriculum por competencias. Una aproximación desde el modelo sistémico complejo (pp. 17-39). Lima, A.B.: Representaciones Generales S.R.L.

González Geraldo, J.L., del Rincón Igea, B., Bonilla Sánchez, A.A. y Sáez López, J.M. (2012). Aprendizajes colaterales: límites y retos del aprendizaje por competencias. Ensayos Revista de la Facultad de Educación de Albacete, 27, 189-202. Recuperado de http://www.revista.uclm.es/index.php/ensayos/article/view/247

Le Boterf, G. (1993). Cómo gestionar la calidad de la formación. Barcelona: Aedipe.

Maroco, J. y García-Marques, T. (2006). Qual a fiabilidade do alfa de Cronbach? Questões antigas e soluções modernas? Laboratórico de Psicologia,4(1), 65-90. 
Martín, X. y Puig, J.M. (2007). Las siete competencias básicas para educar en valores. Barcelona: Grao

McMillan, J. y Schumacher, S. (2005). Investigación educativa. Madrid: Pearson.

Ministerio de Educación, Cultura y Deporte (MECD) (2003). La integración del sistema universitario en el Espacio Europeo de Educación Superior. Documento-Marco. Madrid: Autor.

Montero, M. (2010). El proceso de Bolonia y las nuevas competencias. Tejuelo, 9, 19-37.

Mora, J.G. (2003). Competencias y empleo de los jóvenes graduados universitarios.Revista de Educación,330,157-170.

Muñoz Cantero, J.M., Rebollo Quintela, N. y Espiñeira Bellón, E.M. (2013). Desarrollo, dominio y relevancia de competencias en el Grado en Educación Social. Revista Galego-Portuguesa de Psicoloxía e Educación, 21(1), 228-229.

Pazo Haro, C.I. y Tejada Mora, J. (2012). Las competencias profesionales en Educación Física. Retos. Nuevas tendencias en Educación Física, Deporte y Recreación,22,5-8.

Pavié, A. (2011). Formación docente: hacia una definición del concepto de competencia profesional docente. REIFOP, 14 (1), 67-80.

Proyecto Tuning (2003). Tuning Educational Structures in Europe. Informe final. Proyecto piloto. Fase 1. Bilbao: Universidad de Deusto.

Riesco, M. (2008). El enfoque por competencias en el EEES y sus implicaciones en la enseñanza y el aprendizaje. Tendencias Pedagógicas, 13, 79-105.

Sobrado, L. (2010). Competencias (profesionales, académicas). En J. A. Caride Gómez y F. Trillo Alonso (Eds.), Dicccionario Galego de Psicopedagoxía (p.126). Santiago de Compostela: Galaxia. 


\section{Autores}

Jesús Miguel Muñoz Cantero

Profesor Titular del Área de Métodos de Investigación y Diagnóstico en Educación. Desarrolla su actividad investigadora en el campo de la evaluación, atención a la diversidad, calidad educativa y acreditación en el marco de la enseñanza universitaria como no universitaria.

Universidad de A Coruña (UDC). Facultad de Ciencias de la Educación, Campus de Elviña, s/n. CP 15071, A Coruña. Tfno.: 981 167000, ext. 1799. Fax: 981167153. jesus.miguel.munoz@udc.es

Nuria Rebollo Quintela

Profesora Contratada con cargo al Plan 12C de la Xunta de Galicia en el Área de Métodos de Investigación y Diagnóstico en Educación. Desarrolla su actividad investigadora en análisis y evaluación de las políticas del mercado laboral en colectivos con especiales dificultades de acceso al empleo, asimismo también trabaja la evaluación en el Espacio Europeo de Educación Superior.

Universidad de A Coruña (UDC). Facultad de Ciencias de la Educación, Campus de Elviña, s/n. CP 15071, A Coruña.Tfno.: 981 167000, ext. 1816. Fax: 981 167153.nuria.rebollo@udc.es

\section{Eva María Espiñeira Bellón}

Doctora por la Universidad de A Coruña en Psicopedagogía (2008). Profesora del Área de Métodos de Investigación en Educación de la Universidad de A Coruña. Desarrolla su actividad investigadora en el campo de la evaluación, calidad educativa, atención a la diversidad e inclusión socioeducativa

Universidad de A Coruña (UDC). Facultad de Ciencias de la Educación, Campus de Elviña, s/n. CP 15071, A Coruña.Tfno.: 981 167000, ext. 1891. Fax: 981 167153. eva.espineira@udc.es 


\section{Anexos}

Tabla 2.Análisis descriptivo y Friedman de las competencias nucleares Educación Primaria

\begin{tabular}{|c|c|c|c|c|}
\hline Competencias nucleares & Dimensión & $\bar{x}$ & S & Sig \\
\hline \multirow{3}{*}{$\begin{array}{l}\text { Expresarse correctamente, tanto de forma oral como escrita, } \\
\text { en las lenguas oficiales de la comunidad autónoma }\end{array}$} & Desarrollo & 3,42 & 1,31 & \multirow[t]{3}{*}{$X^{2}=54,51^{*}$} \\
\hline & Dominio & 4,05 & ,89 & \\
\hline & Relevancia & 4,78 & ,62 & \\
\hline \multirow{3}{*}{$\begin{array}{l}\text { Dominar la expresión y la comprensión de forma oral y escrita } \\
\text { de un idioma extranjero }\end{array}$} & Desarrollo & 2,35 & 1,07 & \multirow[t]{3}{*}{$X^{2}=74,22^{*}$} \\
\hline & Dominio & 3,07 & 1,27 & \\
\hline & Relevancia & 4,6 & ,76 & \\
\hline \multirow{3}{*}{$\begin{array}{l}\text { Utilizar las herramientas básicas de las TIC necesarias para el } \\
\text { ejercicio de su profesión y para el aprendizaje a lo largo de su } \\
\text { vida }\end{array}$} & Desarrollo & 2,95 & 1,19 & \multirow[t]{3}{*}{$X^{2}=55,39^{*}$} \\
\hline & Dominio & 3,73 & 1,11 & \\
\hline & Relevancia & 4,58 & ,74 & \\
\hline \multirow{3}{*}{$\begin{array}{l}\text { Desarrollarse para el ejercicio de una ciudadanía abierta [...] } \\
\text { capaz de analizar la realidad, diagnosticar problemas, formular } \\
\text { e implantar soluciones basadas en el conocimiento y } \\
\text { orientadas al bien común }\end{array}$} & Desarrollo & 2,82 & 1,18 & \multirow[t]{3}{*}{$X^{2}=65,30^{*}$} \\
\hline & Dominio & 3,68 & ,85 & \\
\hline & Relevancia & 4,58 & ,84 & \\
\hline \multirow{3}{*}{$\begin{array}{l}\text { Entender la importancia de la cultura emprendedora y conocer } \\
\text { los medios al alcance de las personas emprendedoras }\end{array}$} & Desarrollo & 2,15 & 1,02 & \multirow[t]{3}{*}{$X^{2}=73,06^{*}$} \\
\hline & Dominio & 3,12 & 1,00 & \\
\hline & Relevancia & 4,10 & 98 & \\
\hline \multirow{3}{*}{$\begin{array}{l}\text { Valorar críticamente el conocimiento, la tecnología y la } \\
\text { información disponible para resolver los problemas con los } \\
\text { que deben enfrentarse }\end{array}$} & Desarrollo & 2,71 & 1,19 & \multirow[t]{3}{*}{$X^{2}=58,27^{*}$} \\
\hline & Dominio & 3,37 & ,99 & \\
\hline & Relevancia & 4,28 & 877 & \\
\hline \multirow{3}{*}{$\begin{array}{l}\text { Asumir como profesional y ciudadano la importancia del } \\
\text { aprendizaje a lo largo de la vida }\end{array}$} & Desarrollo & 3,55 & 1,33 & \multirow[t]{3}{*}{$X^{2}=39,53^{*}$} \\
\hline & Dominio & 4,27 & 80 & \\
\hline & Relevancia & 4,73 &, 50 & \\
\hline \multirow{3}{*}{$\begin{array}{l}\text { Valorar la importancia que tiene la investigación, la innovación } \\
\text { y el desarrollo tecnológico en el avance socioeconómico y } \\
\text { cultural de la sociedad }\end{array}$} & Desarrollo & 2,95 & 1,09 & \multirow[t]{3}{*}{$X^{2}=53,78^{*}$} \\
\hline & Dominio & 3,61 & 99 & \\
\hline & Relevancia & 4,50 & ,641 & \\
\hline
\end{tabular}

El nivel de significancia es .001

Tabla 3.Análisis descriptivo y Friedman de las competencias específicas Educación Primaria

\begin{tabular}{|c|c|c|c|c|}
\hline Competencias específicas & Dimensión & $\overline{\mathrm{x}}$ & S & Sig \\
\hline \multirow{3}{*}{$\begin{array}{l}\text { Comprender los procesos de aprendizaje relativos al período } \\
\text { de } 6-12 \text {, en el contexto familiar, social y escolar }\end{array}$} & Desarrollo & 3,42 & ,76 & \multirow[t]{3}{*}{$X^{2}=80,35^{*}$} \\
\hline & Dominio & 3,20 & 84 & \\
\hline & Relevancia & 4,80 & ,46 & \\
\hline \multirow{3}{*}{$\begin{array}{l}\text { Conocer las características de estos estudiantes, así como las } \\
\text { características de sus contextos motivacionales y sociales }\end{array}$} & Desarrollo & 3,29 & ,95 & \multirow[t]{3}{*}{$X^{2}=85,14^{*}$} \\
\hline & Dominio & 3,34 & 91 & \\
\hline & Relevancia & 4,85 &, 53 & \\
\hline \multirow{3}{*}{$\begin{array}{l}\text { Dominar los conocimientos necesarios para comprender el } \\
\text { desarrollo de la personalidad de estos estudiantes e } \\
\text { identificar disfunciones }\end{array}$} & Desarrollo & 2,69 & 96 & \multirow[t]{3}{*}{$X^{2}=82,81^{*}$} \\
\hline & Dominio & 2,80 &, 78 & \\
\hline & Relevancia & 4,73 & ,72 & \\
\hline \multirow{3}{*}{$\begin{array}{l}\text { Identificar dificultades de aprendizaje, informarlas y colaborar } \\
\text { en su tratamiento }\end{array}$} & Desarrollo & 2,80 & 1,13 & \multirow[t]{3}{*}{$X^{2}=84,63^{*}$} \\
\hline & Dominio & 3,00 & 1,07 & \\
\hline & Relevancia & 4,88 & 46 & \\
\hline \multirow{3}{*}{$\begin{array}{l}\text { Conocer las propuestas y desarrollos actuales basado en el } \\
\text { aprendizaje de competencias }\end{array}$} & Desarrollo & 3,25 & ,91 & \multirow[t]{3}{*}{$X^{2}=45,47^{*}$} \\
\hline & Dominio & 2,98 & ,93 & \\
\hline & Relevancia & 4,08 & ,73 & \\
\hline Identificar y planificar la resolución de situaciones educativas & Desarrollo & 2,60 & 1,12 & $X^{2}=75,92^{*}$ \\
\hline
\end{tabular}




\begin{tabular}{|c|c|c|c|c|}
\hline Competencias específicas & Dimensión & $\overline{\mathrm{x}}$ & $\mathrm{S}$ & Sig \\
\hline \multirow{2}{*}{$\begin{array}{l}\text { que afectan a estudiantes con diferentes capacidades y } \\
\text { distintos ritmos de aprendizaje }\end{array}$} & Dominio & 2,90 & 1,04 & \\
\hline & Relevancia & 4,75 & ,81 & \\
\hline \multirow{3}{*}{$\begin{array}{l}\text { Analizar y comprender los procesos educativos en el aula y } \\
\text { fuera de ella relativos al periodo 6-12 }\end{array}$} & Desarrollo & 3,04 & ,88 & \multirow[t]{3}{*}{$X^{2}=69,08^{*}$} \\
\hline & Dominio & 3,02 & 1,08 & \\
\hline & Relevancia & 4,38 & ,84 & \\
\hline \multirow{3}{*}{ Conocer los fundamentos de la educación primaria } & Desarrollo & 3,62 & 1,03 & \multirow[t]{3}{*}{$X^{2}=22,04^{*}$} \\
\hline & Dominio & 3,49 & ,95 & \\
\hline & Relevancia & 4,20 & 85 & \\
\hline \multirow{3}{*}{$\begin{array}{l}\text { Analizar la práctica docente y las condiciones institucionales } \\
\text { que la enmarcan }\end{array}$} & Desarrollo & 3,20 & 1,03 & \multirow[t]{3}{*}{$X^{2}=32,91^{*}$} \\
\hline & Dominio & 3,22 & ,88 & \\
\hline & Relevancia & 4,03 & 1,00 & \\
\hline \multirow{3}{*}{$\begin{array}{l}\text { Conocer la evolución histórica del sistema educativo en } \\
\text { nuestro país y los condicionantes políticos y legislativos de la } \\
\text { actividad educativa }\end{array}$} & Desarrollo & 4,04 & ,77 & \multirow[t]{3}{*}{$X^{2}=22,98^{*}$} \\
\hline & Dominio & 3,56 & 1,02 & \\
\hline & Relevancia & 3,60 & ,98 & \\
\hline \multirow[t]{3}{*}{ Conocer los procesos de interacción y comunicación en el aula } & Desarrollo & 3,16 & 1,15 & \multirow[t]{3}{*}{$X^{2}=70,29^{*}$} \\
\hline & Dominio & 3,22 & 1,06 & \\
\hline & Relevancia & 4,53 &, 71 & \\
\hline \multirow[t]{3}{*}{ Abordar y resolver problemas de disciplina } & Desarrollo & 2,09 & 1,08 & \multirow[t]{3}{*}{$X^{2}=82,70^{*}$} \\
\hline & Dominio & 2,63 & ,94 & \\
\hline & Relevancia & 4,55 &, 71 & \\
\hline \multirow{3}{*}{$\begin{array}{l}\text { Promover el trabajo cooperativo y el trabajo y esfuerzo } \\
\text { individuales }\end{array}$} & Desarrollo & 3,60 & 1,04 & \multirow[t]{3}{*}{$X^{2}=42,15^{*}$} \\
\hline & Dominio & 3,68 & ,93 & \\
\hline & Relevancia & 4,48 & 71 & \\
\hline \multirow{3}{*}{$\begin{array}{l}\text { Promover acciones de educación en valores orientadas a la } \\
\text { preparación de una ciudadanía activa y democrática }\end{array}$} & Desarrollo & 3,07 & 1,13 & \multirow[t]{3}{*}{$X^{2}=61,85^{*}$} \\
\hline & Dominio & 3,41 & 1,05 & \\
\hline & Relevancia & 4,48 &, 75 & \\
\hline \multirow{3}{*}{$\begin{array}{l}\text { Conocer y abordar situaciones escolares en contextos } \\
\text { multiculturales }\end{array}$} & Desarrollo & 3,04 & 1,11 & \multirow[t]{3}{*}{$X^{2}=66,66^{*}$} \\
\hline & Dominio & 3,20 & ,95 & \\
\hline & Relevancia & 4,53 &, 72 & \\
\hline Diseñar, planificar y evaluar la actividad docente y el & Desarrollo & 3,71 & 1,01 & $X^{2}=43,26^{*}$ \\
\hline aprendizaje en el aula & Dominio & 3,63 & ,88 & \\
\hline & Relevancia & 4,63 &, 58 & \\
\hline Conocer y aplicar experiencias innovadoras en educación & Desarrollo & 3,02 & 1,04 & $X^{2}=58,17^{*}$ \\
\hline primaria & Dominio & 3,02 & ,96 & \\
\hline & Relevancia & 4,50 & ,87 & \\
\hline Participar en la definición del proyecto educativo y en la & Desarrollo & 2,29 & 1,10 & $X^{2}=64,64^{*}$ \\
\hline actividad general del centro atendiendo a criterios de gestión & Dominio & 2,29 & 1,03 & \\
\hline & Relevancia & 3,78 & ,97 & \\
\hline Conocer y aplicar metodologías y técnicas básicas de & Desarrollo & 2,29 & 1,13 & $X^{2}=75,06^{*}$ \\
\hline $\begin{array}{l}\text { investigación educativa y ser capaz de diseñar proyectos de } \\
\text { innovación identificando indicadores de evaluación }\end{array}$ & Dominio & 2,37 & 1,11 & \\
\hline & Relevancia & 3,88 & 1,04 & \\
\hline Mostrar habilidades sociales para entender a las familias y & Desarrollo & 1,98 & 1,15 & $X^{2}=76,38^{*}$ \\
\hline hacerse entender por ellas & Dominio & 2,49 & 1,18 & \\
\hline & Relevancia & 4,45 &, 75 & \\
\hline Conocer y saber ejercer las funciones de tutor y orientador en & Desarrollo & 2,80 & 1,22 & $X^{2}=66,79^{*}$ \\
\hline relación con la educación familiar en el periodo 6-12 & Dominio & 2,76 & 1,20 & \\
\hline & Relevancia & 4,48 & ,78 & \\
\hline Relacionar la educación con el medio, y cooperar con las & Desarrollo & 2,64 & 1,26 & $X^{2}=66,57^{*}$ \\
\hline familias y la comunidad & Dominio & 2,73 & 1,21 & \\
\hline & Relevancia & 4,40 &, 81 & \\
\hline Analizar e incorporar de forma crítica las cuestiones más & Desarrollo & 2,85 & 1,08 & $X^{2}=72,92^{*}$ \\
\hline relevantes de la sociedad actual que afectan a la educación & Dominio & 3,00 & 1,20 & \\
\hline familiar y escolar $[\ldots]$ & Relevancia & 4,58 &, 71 & \\
\hline Conocer la evolución histórica de la familia, los diferentes & Desarrollo & 2,22 & 1,24 & $X^{2}=70,81^{*}$ \\
\hline
\end{tabular}




\begin{tabular}{|c|c|c|c|c|}
\hline Competencias específicas & Dimensión & $\overline{\mathrm{x}}$ & $\mathrm{S}$ & Sig \\
\hline \multirow{2}{*}{$\begin{array}{l}\text { tipos de familias, de estilos de vida y educación en el contexto } \\
\text { familiar }\end{array}$} & Dominio & 2,63 & 1,29 & \\
\hline & Relevancia & 4,10 & 1,01 & \\
\hline \multirow{3}{*}{$\begin{array}{l}\text { Comprender los principios básicos y las leyes fundamentales } \\
\text { de las ciencias experimentales (Física, Química, Biología y } \\
\text { Geología) }\end{array}$} & Desarrollo & 2,91 & 1,31 & $X^{2}=29,46^{*}$ \\
\hline & Dominio & 2,93 & 1,10 & \\
\hline & Relevancia & 3,73 & 1,15 & \\
\hline \multirow[t]{3}{*}{ Conocer el currículo escolar de estas ciencias } & Desarrollo & 3,37 & 1,10 & $X^{2}=13,74^{*}$ \\
\hline & Dominio & 3,37 & 1,09 & \\
\hline & Relevancia & 3,88 & ,96 & \\
\hline \multirow{3}{*}{$\begin{array}{l}\text { Plantear y resolver problemas asociados con las ciencias a la } \\
\text { vida cotidiana }\end{array}$} & Desarrollo & 3,50 & 1,05 & $X^{2}=43,96^{*}$ \\
\hline & Dominio & 3,49 & 1,12 & \\
\hline & Relevancia & 4,23 & ,92 & \\
\hline \multirow[t]{3}{*}{ Valorar las ciencias como un hecho cultural } & Desarrollo & 3,11 & 1,14 & $X^{2}=38,94^{*}$ \\
\hline & Dominio & 3,17 & 1,09 & \\
\hline & Relevancia & 4,00 & ,96 & \\
\hline \multirow{3}{*}{$\begin{array}{l}\text { Reconocer la mutua influencia entre ciencia, sociedad y } \\
\text { desarrollo tecnológico, así como las conductas ciudadanas } \\
\text { pertinentes, para procurar un futuro sostenible }\end{array}$} & Desarrollo & 3,24 & 1,15 & $X^{2}=33,17^{*}$ \\
\hline & Dominio & 3,39 & 1,07 & \\
\hline & Relevancia & 4,13 & 1,04 & \\
\hline \multirow{3}{*}{$\begin{array}{l}\text { Desarrollar y evaluar contenidos del currículo mediante } \\
\text { recursos didácticos apropiados y promover la adquisición de } \\
\text { competencias básicas en los estudiantes }\end{array}$} & Desarrollo & 3,45 & 1,03 & $X^{2}=48,28^{*}$ \\
\hline & Dominio & 3,39 & 1,02 & \\
\hline & Relevancia & 4,38 & ,92 & \\
\hline \multirow[t]{3}{*}{ Comprender los principios básicos de las ciencias sociales } & Desarrollo & 3,15 & 1,01 & $X^{2}=36,11^{*}$ \\
\hline & Dominio & 3,17 & 1,09 & \\
\hline & Relevancia & 4,03 & ,95 & \\
\hline \multirow[t]{3}{*}{ Conocer el currículo escolar de las ciencias sociales } & Desarrollo & 3,04 & 1,13 & $X^{2}=29,21^{*}$ \\
\hline & Dominio & 3,20 & 1,18 & \\
\hline & Relevancia & 3,98 & ,97 & \\
\hline \multirow{3}{*}{$\begin{array}{l}\text { Integrar el estudio histórico y geográfico desde una } \\
\text { orientación instructiva y cultural }\end{array}$} & Desarrollo & 3,16 & 1,18 & $X^{2}=40,11^{*}$ \\
\hline & Dominio & 3,17 & ,99 & \\
\hline & Relevancia & 4,13 & 1,02 & \\
\hline \multirow{3}{*}{$\begin{array}{l}\text { Fomentar la educación democrática de la ciudadanía y la } \\
\text { práctica del pensamiento social crítico }\end{array}$} & Desarrollo & 3,07 & 1,21 & $X^{2}=60,76^{*}$ \\
\hline & Dominio & 3,44 & 1,23 & \\
\hline & Relevancia & 4,40 & ,95 & \\
\hline \multirow{3}{*}{$\begin{array}{l}\text { Valorar la relevancia de las instituciones públicas y privadas } \\
\text { para la convivencia pacífica entre los pueblos }\end{array}$} & Desarrollo & 2,44 & 1,31 & $X^{2}=41,74^{*}$ \\
\hline & Dominio & 2,90 & 1,26 & \\
\hline & Relevancia & 3,65 & 1,09 & \\
\hline \multirow{3}{*}{$\begin{array}{l}\text { Conocer el hecho religioso a lo largo de la historia y su } \\
\text { relación con la cultura }\end{array}$} & Desarrollo & 2,44 & 1,33 & $X^{2}=16,94^{*}$ \\
\hline & Dominio & 2,71 & 1,35 & \\
\hline & Relevancia & 3,20 & 1,24 & \\
\hline \multirow{3}{*}{$\begin{array}{l}\text { Desarrollar y evaluar contenidos del currículo mediante } \\
\text { recursos didácticos apropiados y promover las competencias } \\
\text { correspondientes en los estudiantes }\end{array}$} & Desarrollo & 3,25 &, 91 & $X^{2}=60,81^{*}$ \\
\hline & Dominio & 3,34 & ,94 & \\
\hline & Relevancia & 4,25 & 87 & \\
\hline \multirow{3}{*}{$\begin{array}{l}\text { Adquirir competencias matemáticas básicas (numéricas, } \\
\text { cálculo, geométricas, representaciones espaciales, estimación } \\
\text { y medida, organización e interpretación de la información, } \\
\text { etc.) }\end{array}$} & Desarrollo & 3,91 & 1,09 & $X^{2}=25,33^{*}$ \\
\hline & Dominio & 3,63 & 1,09 & \\
\hline & Relevancia & 4,50 & 87 & \\
\hline \multirow{3}{*}{$\begin{array}{l}\text { Conocer el currículo escolar de matemáticas. Analizar, razonar } \\
\text { y comunicar propuestas matemáticas }\end{array}$} & Desarrollo & 3,85 & 1,13 & $X^{2}=16,55^{*}$ \\
\hline & Dominio & 3,63 & 1,24 & \\
\hline & Relevancia & 4,48 &, 72 & \\
\hline \multirow[t]{3}{*}{ Plantear y resolver problemas vinculados con la vida cotidiana } & Desarrollo & 3,62 & 1,15 & $X^{2}=58,15^{*}$ \\
\hline & Dominio & 3,54 & 1,07 & \\
\hline & Relevancia & 4,33 & ,88 & \\
\hline
\end{tabular}




\begin{tabular}{|c|c|c|c|c|}
\hline Competencias específicas & Dimensión & $\overline{\mathrm{x}}$ & $\mathrm{S}$ & Sig \\
\hline \multirow{3}{*}{$\begin{array}{l}\text { Valorar la relación entre matemáticas y ciencias como uno de } \\
\text { los pilares del pensamiento científico }\end{array}$} & Desarrollo & 3,13 & 1,09 & \multirow[t]{3}{*}{$X^{2}=43,13^{*}$} \\
\hline & Dominio & 3,22 & 1,06 & \\
\hline & Relevancia & 3,95 & 1,04 & \\
\hline \multirow{3}{*}{$\begin{array}{l}\text { Desarrollar y evaluar contenidos del currículo mediante } \\
\text { recursos didácticos apropiados y promover las competencias } \\
\text { correspondientes en los estudiantes }\end{array}$} & Desarrollo & 3,33 & 1,02 & \multirow[t]{3}{*}{$X^{2}=54,33^{*}$} \\
\hline & Dominio & 3,46 & 1,03 & \\
\hline & Relevancia & 4,30 &, 85 & \\
\hline \multirow{3}{*}{$\begin{array}{l}\text { Comprender los principios básicos de las ciencias del lenguaje } \\
\text { y la comunicación }\end{array}$} & Desarrollo & 2,91 & 1,13 & \multirow[t]{3}{*}{$X^{2}=61,92^{*}$} \\
\hline & Dominio & 3,22 & 1,04 & \\
\hline & Relevancia & 4,18 & ,98 & \\
\hline \multirow[t]{3}{*}{ Adquirir formación literaria y conocer la literatura infantil } & Desarrollo & 3,15 & 1,25 & \multirow[t]{3}{*}{$X^{2}=47,36^{*}$} \\
\hline & Dominio & 3,17 & 1,18 & \\
\hline & Relevancia & 4,30 & ,96 & \\
\hline \multirow[t]{3}{*}{ Conocer el currículo escolar de las lenguas y la literatura } & Desarrollo & 3,06 & 1,25 & \multirow[t]{3}{*}{$X^{2}=41,67^{*}$} \\
\hline & Dominio & 3,20 & 1,05 & \\
\hline & Relevancia & 4,10 & 1,17 & \\
\hline \multirow{3}{*}{$\begin{array}{l}\text { Hablar, leer y escribir correcta y adecuadamente en las } \\
\text { lenguas oficiales de la Comunidad Autónoma }\end{array}$} & Desarrollo & 3,55 & 1,28 & \multirow{3}{*}{$X^{2}=40,55^{*}$} \\
\hline & Dominio & 2,85 & 1,08 & \\
\hline & Relevancia & 4,70 &, 72 & \\
\hline \multirow{3}{*}{$\begin{array}{l}\text { Conocer el proceso de aprendizaje del lenguaje escrito y su } \\
\text { enseñanza }\end{array}$} & Desarrollo & 2,38 & 1,25 & \multirow[t]{3}{*}{$X^{2}=66,59^{*}$} \\
\hline & Dominio & 3,98 & 1,15 & \\
\hline & Relevancia & 4,58 & ,93 & \\
\hline \multirow[t]{3}{*}{ Fomentar la lectura y animar a escribir } & Desarrollo & 2,58 & 1,34 & \multirow[t]{3}{*}{$X^{2}=67,53^{*}$} \\
\hline & Dominio & 3,59 & 1,28 & \\
\hline & Relevancia & 4,68 &, 83 & \\
\hline \multirow{3}{*}{$\begin{array}{l}\text { Conocer las dificultades para el aprendizaje de las lenguas } \\
\text { oficiales de estudiantes de otras lenguas }\end{array}$} & Desarrollo & 1,91 & 1,09 & \multirow[t]{3}{*}{$X^{2}=81,95^{*}$} \\
\hline & Dominio & 2,41 & 1,18 & \\
\hline & Relevancia & 4,33 & 1,05 & \\
\hline \multirow{3}{*}{$\begin{array}{l}\text { Afrontar situaciones de aprendizaje de lenguas en contextos } \\
\text { multilingües }\end{array}$} & Desarrollo & 1,80 & 1,01 & $X^{2}=$ \\
\hline & Dominio & 2,32 & 1,05 & \\
\hline & Relevancia & 4,28 & 1,08 & \\
\hline Expresarse, oralmente y por escrito en una lengua extranjera & Desarrollo & 2,41 & 1,21 & $X^{2}=66,54^{*}$ \\
\hline & Dominio & 2,80 & 1,28 & \\
\hline & Relevancia & 4,48 & ,93 & \\
\hline Desarrollar y evaluar contenidos del currículo mediante & Desarrollo & 3,13 & 1,09 & $X^{2}=40,82^{*}$ \\
\hline $\begin{array}{l}\text { recursos didácticos apropiados y promover las competencias } \\
\text { correspondientes a los estudiantes }\end{array}$ & Dominio & 3,27 & 1,18 & \\
\hline & Relevancia & 4,83 & 4,51 & \\
\hline Comprender los principios que contribuyen a la formación & Desarrollo & 2,58 & 1,12 & $X^{2}=51,27^{*}$ \\
\hline cultural, personal y social desde las artes & Dominio & 2,83 & 1,09 & \\
\hline & Relevancia & 3,90 & 1,01 & \\
\hline Conocer el currículo escolar de la educación artística, en sus & Desarrollo & 2,55 & 1,17 & $X^{2}=56,91^{*}$ \\
\hline aspectos plástico, audiovisual y musical & Dominio & 2,88 & 1,38 & \\
\hline & Relevancia & 4,08 & ,99 & \\
\hline Adquirir recursos para fomentar la participación a lo largo de & Desarrollo & 2,38 & 1,08 & $X^{2}=63,05^{*}$ \\
\hline la vida en actividades musicales y plásticas dentro y fuera de la & Dominio & 2,80 & 1,17 & \\
\hline escuela & Relevancia & 4,15 & 1,00 & \\
\hline Comprender los principios que contribuyen a la formación & Desarrollo & 3,47 & 99 & $X^{2}=39,28^{*}$ \\
\hline cultural, personal y social desde la educación física & Dominio & 3,37 & 97 & \\
\hline & Relevancia & 4,28 & ,75 & \\
\hline Conocer el currículo escolar de la educación física & Desarrollo & 3,85 & 1,02 & $X^{2}=21,31^{*}$ \\
\hline & Dominio & 3,63 & 1,04 & \\
\hline & Relevancia & 4,08 & 1,02 & \\
\hline Adquirir recursos para fomentar la participación a lo largo de & Desarrollo & 3,76 & 1,11 & $X^{2}=31,88^{*}$ \\
\hline & Dominio & 3,54 & 1,14 & \\
\hline & Relevancia & 4,43 & ,74 & \\
\hline
\end{tabular}




\begin{tabular}{|c|c|c|c|c|}
\hline Competencias específicas & Dimensión & $\overline{\mathrm{x}}$ & $\mathrm{S}$ & Sig \\
\hline \multirow{3}{*}{$\begin{array}{l}\text { Adquirir un conocimiento práctico del aula y de la gestión de } \\
\text { la misma }\end{array}$} & Desarrollo & 3,05 & 1,31 & \multirow[t]{3}{*}{$X^{2}=66,72^{*}$} \\
\hline & Dominio & 3,54 & 1,07 & \\
\hline & Relevancia & 4,63 & ,74 & \\
\hline \multirow{3}{*}{$\begin{array}{l}\text { Conocer y aplicar los procesos de interacción y comunicación } \\
\text { en el aula y dominar las destrezas y habilidades sociales } \\
\text { necesarias para fomentar un clima de aula que facilite el } \\
\text { aprendizaje y la convivencia }\end{array}$} & Desarrollo & 2,55 & 1,01 & \multirow[t]{3}{*}{$X^{2}=78,00$} \\
\hline & Dominio & 3,22 & ,93 & \\
\hline & Relevancia & 4,58 & ,78 & \\
\hline \multirow{3}{*}{$\begin{array}{l}\text { Controlar y hacer el seguimiento del proceso educativo y en } \\
\text { particular el de enseñanza-aprendizaje mediante el dominio } \\
\text { de las técnicas y estrategias necesarias }\end{array}$} & Desarrollo & 2,73 & ,95 & \multirow[t]{3}{*}{$X^{2}=77,26$} \\
\hline & Dominio & 3,10 & ,97 & \\
\hline & Relevancia & 4,55 & ,71 & \\
\hline \multirow{3}{*}{$\begin{array}{l}\text { Relacionar teoría y práctica con la realidad del aula y del } \\
\text { centro }\end{array}$} & Desarrollo & 2,80 & 1,28 & \multirow[t]{3}{*}{$X^{2}=63,08$} \\
\hline & Dominio & 3,22 & 1,03 & \\
\hline & Relevancia & 4,45 & 87 & \\
\hline \multirow{3}{*}{$\begin{array}{l}\text { Participar en la actividad docente y aprender a saber hacer, } \\
\text { actuando y reflexionando desde la práctica }\end{array}$} & Desarrollo & 2,95 & 1,28 & \multirow[t]{3}{*}{$X^{2}=69,75^{*}$} \\
\hline & Dominio & 3,54 & 1,05 & \\
\hline & Relevancia & 4,73 &, 55 & \\
\hline \multirow{3}{*}{$\begin{array}{l}\text { Participar en las propuestas de mejora en los distintos } \\
\text { ámbitos de actuación que se puedan establecer en un centro }\end{array}$} & Desarrollo & 2,24 & 1,18 & \multirow[t]{3}{*}{$X^{2}=76,87^{*}$} \\
\hline & Dominio & 2,76 & 1,20 & \\
\hline & Relevancia & 4,50 & ,84 & \\
\hline \multirow{3}{*}{$\begin{array}{l}\text { Regular los procesos de interacción y comunicación en grupos } \\
\text { de estudiantes 6-12 años }\end{array}$} & Desarrollo & 2,49 & 1,21 & \multirow[t]{3}{*}{$X^{2}=82,37$} \\
\hline & Dominio & 2,93 & 1,05 & \\
\hline & Relevancia & 4,53 &, 78 & \\
\hline \multirow{3}{*}{$\begin{array}{l}\text { Conocer formas de colaboración con los distintos sectores de } \\
\text { la comunidad educativa y del entorno social }\end{array}$} & Desarrollo & 2,35 & 1,15 & \multirow[t]{3}{*}{$X^{2}=63,56$} \\
\hline & Dominio & 2,88 & ,98 & \\
\hline & Relevancia & 4,28 & 84 & \\
\hline
\end{tabular}

El nivel de significancia es .05

Tabla 4. Análisis descriptivo y Friedman de las competencias transversales Educación Primaria

\begin{tabular}{|c|c|c|c|c|}
\hline Competencias transversales & Dimensión & $\bar{x}$ & $\mathrm{~S}$ & Sig \\
\hline \multirow[t]{3}{*}{ Aprender a aprender } & Desarrollo & 3,15 & 1,17 & \multirow{3}{*}{$X^{2}=75,37^{*}$} \\
\hline & Dominio & 3,49 & 1,05 & \\
\hline & Relevancia & 4,65 & ,70 & \\
\hline \multirow[t]{3}{*}{ Resolver problemas de forma efectiva } & Desarrollo & 2,76 & 1,03 & \multirow[t]{3}{*}{$X^{2}=76,15^{*}$} \\
\hline & Dominio & 3,20 & 90 & \\
\hline & Relevancia & 4,48 & ,90 & \\
\hline \multirow[t]{3}{*}{ Aplicar un pensamiento crítico, lógico y creativo } & Desarrollo & 3,20 & 1,13 & \multirow[t]{3}{*}{$X^{2}=67,92^{*}$} \\
\hline & Dominio & 3,54 & 1,00 & \\
\hline & Relevancia & 4,68 & ,69 & \\
\hline \multirow[t]{3}{*}{ Trabajar de forma autónoma con iniciativa } & Desarrollo & 3,20 & 1,35 & \multirow[t]{3}{*}{$X^{2}=62,72^{*}$} \\
\hline & Dominio & 3,90 &, 97 & \\
\hline & Relevancia & 4,83 &, 59 & \\
\hline \multirow[t]{3}{*}{ Trabajar de forma colaborativa } & Desarrollo & 4,12 & 1,19 & \multirow[t]{3}{*}{$X^{2}=22,40^{*}$} \\
\hline & Dominio & 4,29 & ,95 & \\
\hline & Relevancia & 4,85 & ,42 & \\
\hline \multirow{3}{*}{$\begin{array}{l}\text { Comportarse con ética y responsabilidad social como } \\
\text { ciudadano y como profesional }\end{array}$} & Desarrollo & 3,11 & 1,31 & \multirow[t]{3}{*}{$X^{2}=67,01^{*}$} \\
\hline & Dominio & 4,15 & ,88 & \\
\hline & Relevancia & 4,70 & ,68 & \\
\hline \multirow[t]{3}{*}{ Comunicarse de manera efectiva en un contorno de trabajo } & Desarrollo & 3,02 & 1,42 & \multirow[t]{3}{*}{$X^{2}=55,11^{*}$} \\
\hline & Dominio & 4,07 & ,93 & \\
\hline & Relevancia & 4,63 & ,62 & \\
\hline \multirow{3}{*}{$\begin{array}{l}\text { Capacidad para elaborar discursos coherentes y organizados } \\
\text { lógicamente }\end{array}$} & Desarrollo & 2,93 & 1,21 & \multirow[t]{3}{*}{$X^{2}=76,98^{*}$} \\
\hline & Dominio & 3,78 & ,93 & \\
\hline & Relevancia & 4,75 & 49 & \\
\hline
\end{tabular}




\begin{tabular}{|c|c|c|c|c|}
\hline Competencias transversales & Dimensión & $\bar{x}$ & S & Sig \\
\hline \multirow{3}{*}{$\begin{array}{l}\text { Capacidad para exponer las ideas elaboradas, de forma oral y } \\
\text { en la escrita }\end{array}$} & Desarrollo & 3,35 & 1,29 & \multirow[t]{3}{*}{$X^{2}=59,03$} \\
\hline & Dominio & 3,90 & ,97 & \\
\hline & Relevancia & 4,75 &, 58 & \\
\hline \multirow{3}{*}{$\begin{array}{l}\text { Capacidad de expresión oral y escrita en varias lenguas (al } \\
\text { menos en una lengua extranjera) }\end{array}$} & Desarrollo & 2,35 & 1,15 & \multirow[t]{3}{*}{$X^{2}=74,81^{*}$} \\
\hline & Dominio & 2,98 & 1,21 & \\
\hline & Relevancia & 4,55 &, 96 & \\
\hline \multirow{3}{*}{$\begin{array}{l}\text { Capacidad de comprensión de los distintos códigos } \\
\text { audiovisuales y multimedia y manejo de las herramientas } \\
\text { informáticas }\end{array}$} & Desarrollo & 2,64 & 1,02 & \multirow[t]{3}{*}{$X^{2}=71,94^{*}$} \\
\hline & Dominio & 3,37 & 1,13 & \\
\hline & Relevancia & 4,48 & ,78 & \\
\hline \multirow{3}{*}{$\begin{array}{l}\text { Capacidad de selección, de análisis, de evaluación y de } \\
\text { utilización de distintos recursos en la red y multimedia }\end{array}$} & Desarrollo & 2,84 & 1,24 & \multirow[t]{3}{*}{$X^{2}=61,72^{*}$} \\
\hline & Dominio & 3,51 & ,97 & \\
\hline & Relevancia & 4,58 & ,67 & \\
\hline \multirow[t]{3}{*}{ Lectura e interpretación de imágenes } & Desarrollo & 2,25 & 1,23 & \multirow[t]{3}{*}{$X^{2}=68,95$} \\
\hline & Dominio & 3,22 & 1,08 & \\
\hline & Relevancia & 4,18 &, 84 & \\
\hline \multirow{3}{*}{$\begin{array}{l}\text { Capacidad para trabajar en equipo de forma cooperativa, } \\
\text { para organizar y planificar el trabajo, tomando decisiones y } \\
\text { resolviendo problemas, tanto de forma conjunta como } \\
\text { individual }\end{array}$} & Desarrollo & 3,69 & 1,24 & \multirow[t]{3}{*}{$X^{2}=42,42^{*}$} \\
\hline & Dominio & 4,10 & ,86 & \\
\hline & Relevancia & 4,83 &, 50 & \\
\hline \multirow{3}{*}{$\begin{array}{l}\text { Capacidad para utilizar diversas fuentes de información, } \\
\text { seleccionar, analizar, sintetizar y extraer ideas importantes y } \\
\text { gestionar la información }\end{array}$} & Desarrollo & 3,35 & 1,29 & \multirow[t]{3}{*}{$X^{2}=53,58^{*}$} \\
\hline & Dominio & 4,00 & ,97 & \\
\hline & Relevancia & 4,63 & ,63 & \\
\hline \multirow{3}{*}{$\begin{array}{l}\text { Capacidad crítica y creativa en el análisis, planificación y } \\
\text { realización de tareas, como fruto de un pensamiento flexible } \\
\text { y divergente }\end{array}$} & Desarrollo & 3,15 & 1,15 & \multirow[t]{3}{*}{$X^{2}=62,12^{*}$} \\
\hline & Dominio & 3,76 &, 79 & \\
\hline & Relevancia & 4,50 & ,64 & \\
\hline \multirow{3}{*}{$\begin{array}{l}\text { Capacidad de análisis y de autoevaluación tanto del propio } \\
\text { trabajo como del trabajo en grupo }\end{array}$} & Desarrollo & 3,22 & 1,21 & \multirow[t]{3}{*}{$X^{2}=50,12^{*}$} \\
\hline & Dominio & 3,90 & ,86 & \\
\hline & Relevancia & 4,45 & ,75 & \\
\hline \multirow[t]{3}{*}{ Compromiso ético para el ejercicio de las tareas docentes } & Desarrollo & 2,96 & 1,36 & \multirow[t]{3}{*}{$X^{2}=64,63^{*}$} \\
\hline & Dominio & 3,98 &, 96 & \\
\hline & Relevancia & 4,58 & ,67 & \\
\hline \multirow{3}{*}{$\begin{array}{l}\text { Capacidad de adaptarse a nuevas situaciones en una sociedad } \\
\text { cambiante y plural }\end{array}$} & Desarrollo & 2,78 & 1,17 & \multirow[t]{3}{*}{$X^{2}=78,19^{*}$} \\
\hline & Dominio & 3,78 & ,91 & \\
\hline & Relevancia & 4,68 & ,73 & \\
\hline \multirow[t]{3}{*}{ Relación con diversos interlocutores sociales } & Desarrollo & 2,44 & 1,31 & \multirow[t]{3}{*}{$X^{2}=74,27^{*}$} \\
\hline & Dominio & 3,29 & 87 & \\
\hline & Relevancia & 4,43 & ,71 & \\
\hline
\end{tabular}

El nivel de significancia es .05 